\title{
Exploring the unique function of imprinting control centers in the PWS/AS-responsible region: finding from array-based methylation analysis in cases with variously sized microdeletions
}

Keiko Matsubara 1* Masatsune Itoh², Kenji Shimizu ${ }^{3}$, Shinji Saito ${ }^{4}$, Keisuke Enomoto ${ }^{5,6}$, Kazuhiko Nakabayashi ${ }^{7}$, Kenichiro Hata ${ }^{7}$, Kenji Kurosawa ${ }^{8}$, Tsutomu Ogata ${ }^{1,9}$, Maki Fukami $^{1}$ and Masayo Kagami ${ }^{{ }^{*}}$

\begin{abstract}
Background: Human 15q11-13 is responsible for Prader-Willi syndrome (PWS) and Angelman syndrome (AS) and includes several imprinted genes together with bipartite elements named AS-IC (imprinting center) and PWS-IC. These concertedly confer allele specificity on 15q11-13. Here, we report DNA methylation status of 15q11-13 and other autosomal imprinted differentially methylated regions (iDMRs) in cases with various deletions within the PWS/ AS-responsible region.

Methods: We performed array-based methylation analysis and examined the methylation status of CpG sites in 15q11-13 and in 71 iDMRs in six cases with various microdeletions, eight cases with conventional deletions within 15q11-13, and healthy controls.

Results: We detected 89 CpGs in 15q11-13 showing significant methylation changes in our cases. Of them, 14 CpGs in the SNORD116s cluster presented slight hypomethylation in the PWS cases and hypermethylation in the AS cases. No iDMRs at regions other than 15q11-13 showed abnormal methylation.

Conclusions: We identified CpG sites and regions in which methylation status is regulated by AS-IC and PWS-IC. This result indicated that each IC had unique functions and coordinately regulated the DNA methylation of respective alleles. In addition, only aberrant methylation at iDMRs in 15q11-13 leads to the development of the phenotypes in our cases.
\end{abstract}

Keywords: 15q11-13, Prader-Willi syndrome, Angelman syndrome, Genome-wide methylation study, Deletion

\section{Introduction}

The chromosomal region 15q11-13 includes several genes showing monoallelic expression in a parent-of-origin-specific manner [1]. This region is responsible for Prader-Willi syndrome (PWS, OMIM \#176270) and Angelman syndrome (AS, OMIM \#105830). PWS is characterized by hypotonia during infancy, developmental delay, hyperphagia followed by morbid obesity, and

\footnotetext{
*Correspondence: matsubara-k@ncchd.go.jp; kagami-ms@ncchd.go.jp 1 Department of Molecular Endocrinology, National Center for Child Health and Development, 2-10-1 Ohkura, Setagaya-ku, Tokyo 157-8535, Japan Full list of author information is available at the end of the article
}

cognitive impairment [2], and AS is associated with severe developmental delay, ataxia, and recurrent seizures [3]. Approximately $70 \%$ of PWS and AS patients have 5-7 Mb deletions affecting the 15q11-13 imprinted region (Fig. 1). Recently, it has been reported that deletion involving the SNORD116 gene, which is one of the C/D box small nucleolar RNAs (snoRNAs), causes the phenotypes of PWS [4-8]. AS phenotypes arise from the loss of expression or function of the UBE3A gene, which is expressed on the maternally derived allele in mature neurons [3]. 


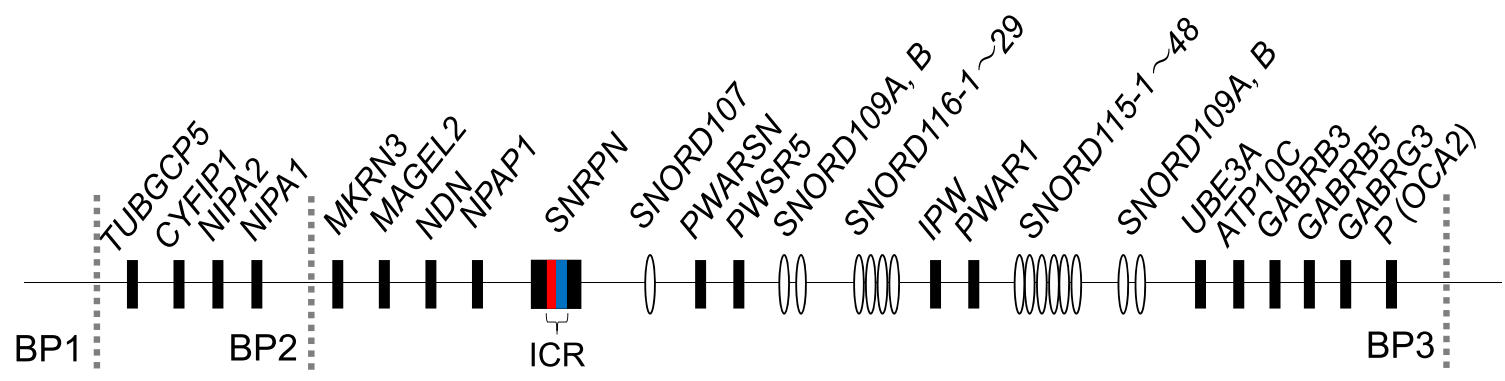

\section{Conventional}

\section{deletion}

Fig. 1 Schematic diagram of the PWS/AS region. Horizontal gray line and dotted line indicate the range of conventional large deletion bounded by breakpoint (BP) 1 or 2 and BP 3. snoRNA genes are shown in ovals. Not all genes of the locus are shown, and the map is not to scale

The imprinting control region (ICR) conferring parent-of-origin identity of the genes on 15q11-13 was defined according to the smallest region of overlap (SRO) found in PWS or AS individuals with rare atypical microdeletions [9]. The ICR on 15q11-13 consists of bipartite DNA elements named AS-IC (imprinting center) and PWS-IC [10]. The PWS-IC is a 4.1-kb region, which spans the SNURF/SNRPN promoter and exon 1, and includes an imprinted differentially methylated region (iDMR), which is maternally methylated and paternally unmethylated. The AS-IC is an 880-bp sequence located $\sim 35 \mathrm{~kb}$ centromeric of the PWS-IC and does not include iDMR. It is known that PWS-IC and AS-IC cooperate in regulating epigenetic status and allele-specific gene expression at this locus [1].

Recent advancement in methylation analysis allows genome-wide methylation studies, and it detected novel iDMRs located in regions near the SNURF/SNRPN gene and several $\mathrm{CpG}$ sites in the SNORD116s cluster showing a slight tendency for preferential paternal methylation [11]. The regulatory mechanism in DNA methylation of those imprinted regions by ICR has not yet been elucidated precisely.

In this study, we performed an array-based DNA methylation analysis in the cases with various deletions involving the PWS/AS region and examined the

Table 1 Clinical information, deletion range, and methylation status at SNRPN-DMR in cases enrolled in this study

\begin{tabular}{|c|c|c|c|c|c|c|}
\hline \multicolumn{2}{|l|}{ Cases } & Phenotype & Sex & $\mathrm{Age}^{\mathrm{a}}$ & Breakpoint (approximate size) & Methylation status at SNRPN-DMR ${ }^{d}$ \\
\hline \multicolumn{2}{|l|}{ Case 1} & PWS & Male & $2 \mathrm{mo}$ & Chr15:25,150,978-25,225,535 (75 kb) & Hypermethylated \\
\hline \multicolumn{2}{|l|}{ Case 2} & Healthy carrier (father of case 1) & Male & $36 \mathrm{yr}$ & & Hypomethylated \\
\hline \multicolumn{2}{|l|}{ Case 3} & PWS & Female & $3 y r$ & Chr15:25,216,569-25,415,670 (200 kb) & Normal \\
\hline \multicolumn{2}{|l|}{ Case 4} & AS & Female & $3 y r$ & Chr15:25,126,774-25,168,037 (41 kb) & Hypomethylated \\
\hline \multicolumn{2}{|l|}{ Case 5} & AS & Male & $4 \mathrm{yr}$ & Chr15:25,164,853-25,168,575 (3.7 kb) & Hypomethylated \\
\hline \multicolumn{2}{|l|}{ Case 6} & Healthy carrier (mother of case 5) & Female & $36 \mathrm{yr}$ & & Normal \\
\hline \multirow[t]{4}{*}{$P W S_{L D}$} & $P W S_{L D^{-1}}$ & PWS & Male & $0 \mathrm{mo}$ & BP2-3 $(5.5 \mathrm{Mb})^{\mathrm{c}}$ & Hypermethylated \\
\hline & $P W S_{L D}-2$ & PWS & Male & $2 \mathrm{mo}$ & $\mathrm{BP} 1-3(6 \mathrm{Mb})^{\mathrm{c}}$ & Hypermethylated \\
\hline & $P W S_{L D}-3$ & PWS & Male & $3 \mathrm{yr}$ & $\mathrm{BP} 1-3(6 \mathrm{Mb})^{\mathrm{c}}$ & Hypermethylated \\
\hline & $P W S_{L D}-4$ & PWS & Male & $8 \mathrm{yr}$ & $\mathrm{BP} 1-3(6 \mathrm{Mb})^{\mathrm{c}}$ & Hypermethylated \\
\hline \multirow[t]{4}{*}{$A S_{L D}$} & $\mathrm{AS}_{\mathrm{LD}}-1$ & AS & Female & $9 \mathrm{yr}$ & BP2-3 $(5.5 \mathrm{Mb})^{\mathrm{C}}$ & Hypomethylated \\
\hline & $\mathrm{AS}_{\mathrm{LD}}-2$ & AS & Female & $9 \mathrm{yr}$ & BP2-3 $(5.5 \mathrm{Mb})^{\mathrm{C}}$ & Hypomethylated \\
\hline & $\mathrm{AS}_{\mathrm{LD}-3}$ & AS & Male & $2 \mathrm{yr}$ & $\mathrm{BP} 1-3(6 \mathrm{Mb})^{\mathrm{c}}$ & Hypomethylated \\
\hline & $\mathrm{AS}_{\mathrm{LD}^{-4}}$ & AS & Female & $1 \mathrm{yr}$ & $\mathrm{BP} 1-3(6 \mathrm{Mb})^{\mathrm{c}}$ & Hypomethylated \\
\hline
\end{tabular}

PWS Prader-Willi syndrome, AS Angelman syndrome, DMR differentially methylated region, $L D$ large deletion, $B P$ breakpoint

${ }^{a}$ Age at sample collection (mo months, yr years)

${ }^{\mathrm{b}}$ The breakpoints were estimated according to the results of aCGH

'The breakpoints were estimated according to the results of methylation-specific multiplex ligation-dependent probe amplification (MS-MLPA). The locations of BPs are shown in Additional file 2: Figure S1

${ }^{\mathrm{d}}$ Methylation status were examined by MS-MLPA 
methylation status of CpG sites in 15q11-13 and in 71 iDMRs at regions other than the PWS/AS region to clarify the regulatory mechanism for DNA methylation in the 15q11-13 imprinted region.

\section{Results and discussion}

Six cases with atypical microdeletions in the 15q11-13 region (cases 1-6) and four with PWS and four with AS due to conventional large deletions (LD) in 15q11-13 (PWS $S_{\mathrm{LD}}$ and $\mathrm{AS}_{\mathrm{LD}}$, respectively) were enrolled in this study (Table 1 and Additional file 1: Supplementary document).

\section{Deletion size and breakpoint}

Custom-built array CGH revealed a copy number loss in each case (Fig. 2a and Additional file 2: Figure S1). This array contained approximately 38,000 probes for $15 \mathrm{q} 11-$ q13 encompassing the imprinted region and $\sim 10,000$ reference probes for other chromosomal regions $(4 \times 180 \mathrm{~K}$ format, Design ID 032112$)$ (Agilent Technologies, Palo Alto, CA, USA). The detailed information of probes contained in this custom-built array is shown in Additional file 3: Table S1. Cases 1 and 2 had a deletion involving both AS-IC and PWS-IC, case 3 had a deletion involving only the SNORD116s cluster, and cases 4-6 had a deletion involving only AS-IC.

Difference in the DNA methylation status between each case with various microdeletions and controls

We focused on the DNA methylation status at CpG sites on autosomal chromosomes in two groups (groups 1 and 2) and extracted methylation data at 1335 probes located in CpG sites at the 15q11-13 PWS/AS region (group 1, Additional file 4: Table S2) and at 863 probes in the iDMRs at regions other than the PWS/AS region (group 2, Additional file 5: Table S3).

\section{CpG sites in 15q11-13 (probes in group 1)}

We identified 89 probes in group 1 showing significant differences in DNA methylation in at least 1 case against normal controls (Fig. 2b and Additional file 6: Table S4). Most probes located in the miR4508-PWS-IC region
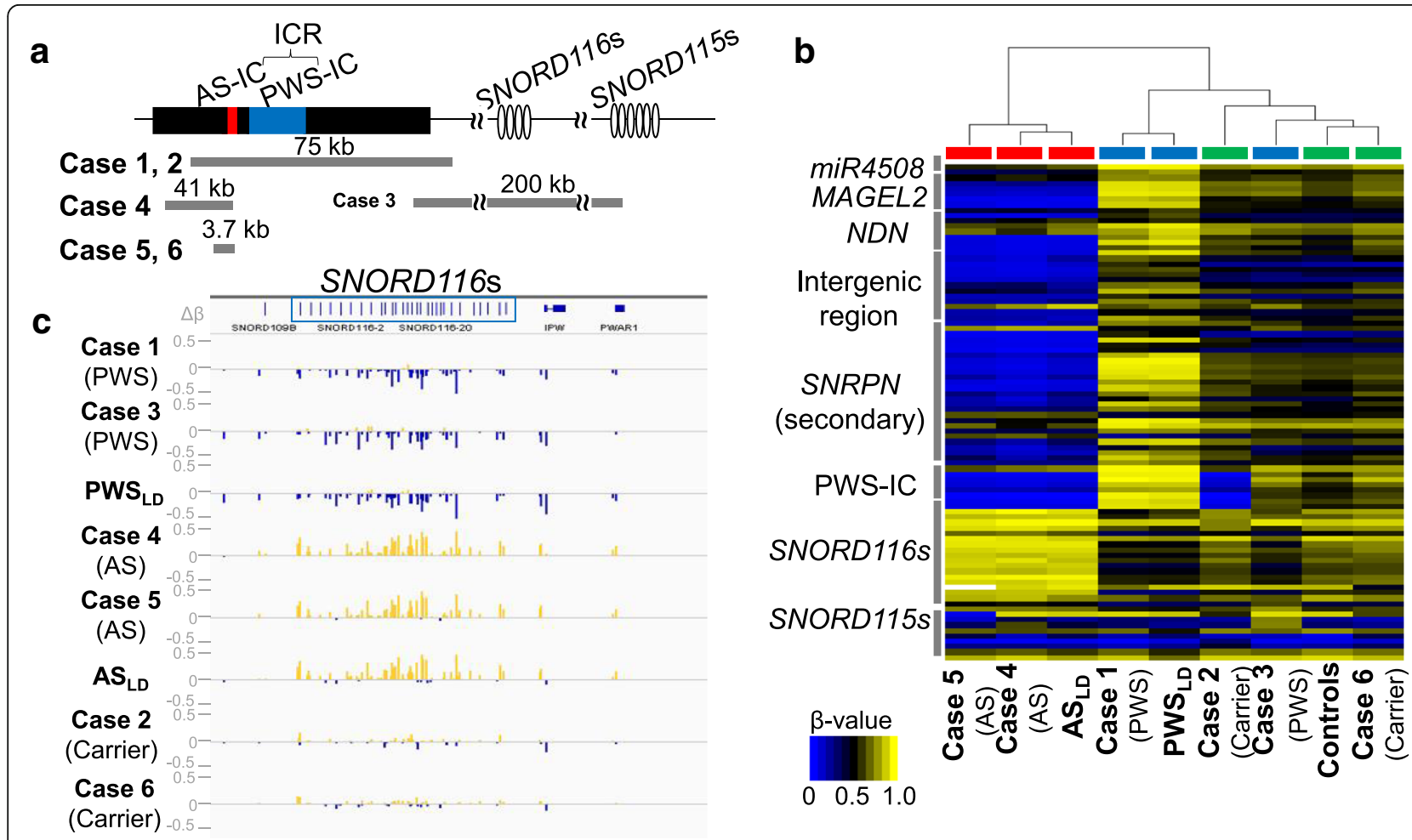

Fig. 2 Genomic organization of the PWS/AS locus and alteration in DNA methylation of CpG sites in 15q11-13. a Summary of the loci and the approximate sizes of the deleted regions in cases 1-6. b Unsupervised hierarchical clustering and heat map of the $450 \mathrm{k}$ methylation data of 89 probes with $|\Delta \beta|>0.2$ and FDR $p$ values $<0.01$ between at least 1 case and controls are shown. The cases with AS phenotype are shown in red boxes, those with PWS phenotype in blue boxes, and the carriers and normal controls in green boxes. Blue and yellow colors indicate 0 and 1 methylation, respectively. The $y$-axis represents the names of iDMRs which had been known to be differentially methylated previously. Not all names of the iDMRs are shown. $\mathbf{c}$ The differences in $\beta$ values $(\Delta \beta)$ of probes located in the SNORD116s and 115s clusters between each case and controls are shown using IGV (Integrated Genome Viewer, http://software.broadinstitute.org/software/igv/). The probes showing "hypermethylated" in cases are represented by yellow vertical bars, and those showing "hypomethylated" by blue ones. ICR, imprinting control region; IC, imprinting center; kb, kilobase 
showed hypermethylation in case 1 and $\mathrm{PWS}_{\mathrm{LD}}$ and hypomethylation in cases 4 and 5 and $\mathrm{AS}_{\mathrm{LD}}$, respectively. Case 3 showed normal methylation status in these sites. Hierarchical clustering of the methylation status of these 89 CpGs showed that all cases other than case 3 with the SNORD116s deletion were classified into subgroups according to their phenotypes (Fig. 2b). Case 3 was clustered closer to the normal controls.

In case 2, who was the father of case 1, probes in his deleted region in PWS-IC showed hypomethylation; however, the remaining probes showed normal methylation status. In case 6 , who was the mother of case 5 , no CpG with aberrant methylation was found.

In addition, 14 probes in the SNORD116s cluster presented slight hypomethylation in the cases with PWS phenotype (cases 1and 3 and $\mathrm{PWS}_{\mathrm{LD}}$ ) and hypermethylation in the cases with AS phenotype (cases 4 and 5 and $\mathrm{AS}_{\mathrm{LD}}$ ) (Fig. 2c).

\section{iDMRs at regions other than the PWS/AS region (probes in group 2)}

Additional file 7: Table S5 shows the results of the methylation analyses for 71 iDMRs including 828 probes in group 2 between each of cases 1-6 and normal controls. We detected no iDMR with an abnormal methylation pattern.

In this study, we examined the methylation status of CpG sites in 15q11-13 and in 71 iDMRs at regions other than the PWS/AS region using methylation array for the cases with various deletions involving the PWS/ AS region. This study provides several notable findings. First, we clarified CpG sites and regions in which the methylation status was regulated by AS-IC and PWS-IC in 15q11-13. Most abnormally methylated CpG sites in cases with deletions were located within or very close to the regions reported in previous studies [11-13]. Based on the results from our patients and a previously reported PWS patient with a microdeletion involving only PWS-IC, we present a hypothetical model for the regulation of DNA methylation at the 15q11-13 imprinted region in Fig. 3. We suggest regulatory mechanisms of this region as follows: (1) maternal pattern (methylated) at CpGs in the upstream region of the ICR was the default state (see normal state and case 2), (2) AS-IC was required for the methylation of PWS-IC on the maternally derived allele (compare case 6 with cases 4 and 5), (3) the unmethylated PWS-IC led to the unmethylated status of CpGs in the upstream of the ICR even on the maternally derived allele (see cases 4 and 5), and (4) the methylation patterns of several CpGs in the SNORD116s clusters were also regulated by both AS-IC and PWS-IC. Several CpGs in the SNORD116s cluster were preferentially methylated on the paternally inherited allele (Fig. 2c) [11]. The methylation pattern in the
SNORD116s cluster was different between case 1 and the PWS case lacking only PWS-IC [14]. These results indicate that both AS-IC and unmethylated PWS-IC on the paternally derived allele function independently as regulators of the methylation status of $\mathrm{CpGs}$ in the SNORD116s cluster. Furthermore, several CpGs in the SNORD116s cluster were methylated in cases 4 and 5 on the maternally derived allele. This result indicates that unmethylated PWS-IC was needed for the methylation of the CpGs in the SNORD116s cluster independently on the parental origin. In the cases with large deletions involving the entire region of 15q11-13 (Fig. 3c), abnormal methylation patterns were simply due to the loss of the paternally or maternally derived allele in PWS or AS patients, respectively.

Second, this study demonstrated that PWS/AS case with various deletions had normal methylation status in known iDMRs at regions other than 15q11-13. This result indicates that the development of phenotypes in our cases was not caused by aberrant methylation changes at iDMRs at regions other than 15q11-13. In addition, our study and previous studies using other methylation analyses showed a normal methylation pattern at PWS-IC in patients with deletions only including the SNORD116s cluster [4-7]. It remains to be clarified how the deletion of SNORD116s contributes to the development of PWS phenotypes. Previous studies reported several findings regarding long non-coding RNAs (sno-lncRNAs) including SNORD116s: (1) some functional units, 116 host genes $(116 \mathrm{HG})$ and snoRNA-related sno-lncRNAs, were processed from the SNORD116s cluster in human tissues or cells $[15,16]$, (2) Fox-family splicing regulators are bound to sno-lncRNAs and altered splicing patterns of genes are related to neuronal development in human ES cells [17], and (3) there was a Snord116-dependent diurnal rhythmic DNA methylation in the mouse cortex [18]. Thus, the loss of the expression of SNORD116s may lead to PWS-relevant phenotypes, such as abnormalities of energy metabolism and diurnal rhythm $[17,18]$.

It remains to be elucidated how AS-IC and PWS-IC establish the allele-specific methylation patterns in neighboring CpGs. Recently, it was reported that transcripts from an oocyte-specific promoter in AS-IC are needed for the acquisition of maternal DNA methylation patterns in PWS-IC in human oocytes [19]. However, there has apparently been no study examining the direct association between AS-IC and the SNORD116s cluster, although unmethylated PWS-IC on the paternal allele physically interacts with paternally expressed genes in the upstream region of ICR [14]. In addition, how unmethylated PWS-IC establishes paternal methylation patterns at CpGs in the PWS/AS region in cis and how PWS-IC interacts with CpG sites at the SNORD116s cluster on paternally derived alleles remain unknown. 


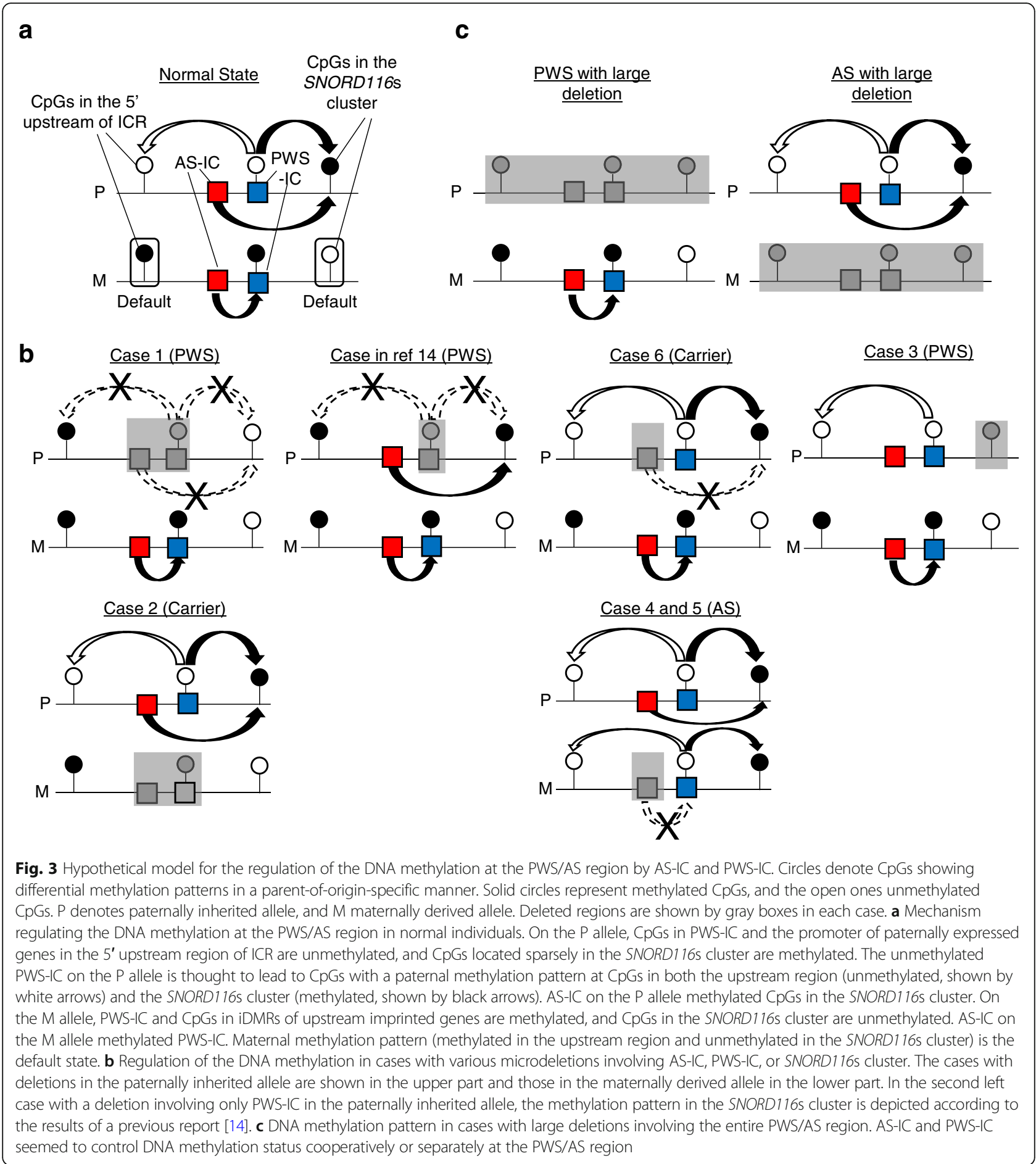

In summary, we performed an array-based methylation study in cases with various-sized microdeletions in the PWS/AS region on maternally or paternally derived chromosome 15 . We identified $\mathrm{CpG}$ sites and regions in which the DNA methylation status is regulated by ICR. Our results enabled us to speculate on the regulatory mechanism for the establishment of the methylation status of local CpG sites or regions in 15q11-13. Moreover, we demonstrated that the development of phenotypes in the PWS/AS patients with deletions encompassing ICR and/or the SNORD116s cluster was not mediated by aberrant methylation changes at iDMRs at regions other than the PWS/AS regions. Further investigation will be 
necessary to fully elucidate how paternal deletion of SNORD116s causes PWS phenotypes.

\section{Materials and methods Subjects}

Six cases with atypical microdeletions in the 15q11-13 region (cases 1-6) and four with $\mathrm{PWS}_{\mathrm{LD}}$ and four with $\mathrm{AS}_{\mathrm{LD}}$ due to conventional large deletions were enrolled in this study (Figs. 1 and 2a, Table 1, Additional file 1: Supplementary document, and Additional file 8: Figure S2). Genomic DNAs from leukocytes in these cases and healthy controls were utilized for this study. Methyl ation-specific multiplex ligation-dependent probe amplification (MS-MLPA; ME030, MRC-Holland, Amsterdam, Netherlands) was performed in all cases to examine the deleted region and DNA methylation status in 15q11-13 (data not shown). All cases showed normal karyotype. Clinical manifestations of cases are shown in the supplementary document. Healthy children $(n=11)$ and adults $(n=24)$ were involved as normal controls.

\section{Copy number analysis}

We designed a custom-built array-based comparative genomic hybridization (aCGH) and utilized this aCGH to narrow down the deleted regions in cases $1-6(4 \times 180 \mathrm{~K}$ format, Design ID 032112, Agilent Technologies, Palo Alto, CA, USA). The detailed information of probes contained in this array is shown in Additional file 3: Table S1. The procedure was as described in the manufacturer's instructions.

\section{Comprehensive methylation analysis using the HumanMethylation450 BeadChip}

We performed methylation assays on cases with various deletions together with healthy controls using the Infinium HumanMethylation450 BeadChip (HM450k, Illumina, Inc., San Diego, CA, USA). Detailed procedures are shown in Additional file 1: Supplementary document.

\section{HM450k data processing}

The HM450k data were processed using the "ChAMP" $\mathrm{R}$ package version 1.10.0 [20]. Detailed algorithms of data pre-processing are available in the supplementary document and Additional file 9: Figure S3. We extracted the methylation data at 1335 probes located in the CpG sites at the 15q11-13 PWS/AS region (group 1, Additional file 4: Table S2) and at 863 probes in the iDMRs at regions other than the PWS/AS region (group 2, Additional file 5: Table S3) [11, 12]. Of note, 161 probes in group 1 presented with allele-specific methylation status according to the parental origin [11-13].

Before making comparisons of the methylation status between each single case with microdeletion (cases 1-6), patients with $\mathrm{PWS}_{\mathrm{LD}}$ or with $\mathrm{AS}_{\mathrm{LD}}$, and controls, we excluded five probes in group 1 (cg26889953, cg1789680, cg09873524, cg26955196, and cg11826104) showing differential methylation status between child and adult controls from the group 1 list for further analysis (Additional file 1: Supplementary document and Additional file 9: Figure S3). There was no probe showing a different methylation status between child and adult controls in group 2.

Subsequently, we compared the methylation status between each single case (cases 1-6), patients with PWS or with AS due to large deletions, and normal controls. The methylation level at each probe was represented by $\beta$ values ranging from 0 (completely unmethylated) to 1 (completely methylated). The differences in DNA methylation $(\Delta \beta)$ were calculated by the subtraction of the $\beta$ value in each case from the average $\beta$ value in controls at each probe site. We considered a probe as differentially methylated when the absolute value of the difference in $\beta$ value $(|\Delta \beta|)$ between two groups was above 0.2 and the false discovery rate (FDR) was below $1 \%$. Detailed methods are available in Additional file 1: Supplementary document.

\section{Additional files}

Additional file 1: Supplementary document. (DOCX $23 \mathrm{~kb}$ )

Additional file 2: Figure S1. Combination of the results of $\mathrm{aCGH}$ and schematic diagram of PWS/AS region. (PPTX $112 \mathrm{~kb}$ )

Additional file 3: Table S1. Probes on a custom-built oligo-microarray. (XLSX $1717 \mathrm{~kb}$ )

Additional file 4: Table S2. Probe list of group 1. (XLSX $102 \mathrm{~kb}$ )

Additional file 5: Table S3. Probe list of group 2. (XLSX 83 kb)

Additional file 6: Table S4. Methylation values of group 1 probes. (XLSX 674 kb)

Additional file 7: Table S5. Methylation values of group 2 probes. (XLSX $459 \mathrm{~kb}$ )

Additional file 8: Figure S2. Family trees of cases with microdeletions enrolled in this study. (PPTX $40 \mathrm{~kb}$ )

Additional file 9: Figure S3. Algorithm for HM450k data processing. (PPTX $46 \mathrm{~kb}$ )

\section{Abbreviations \\ 116HG: 116 host genes; aCGH: Array-based comparative genomic hybridization; AS: Angelman syndrome; HM450k: Infinium HumanMethylation450 BeadChip; IC: Imprinting center; ICR: Imprinting control region; iDMRs: Imprinted differentially methylated regions; LD: Large deletions; MS-MLPA: Methylation-specific multiplex ligation-dependent probe amplification; PWS: Prader-Willi syndrome; sno-IncRNAs: Long non-coding RNAs; SRO: Smallest region of overlap}

\section{Acknowledgements}

We thank Ms. Hiromi Kamura, Tamae Tanji, and Dr. Saki Aoto for their support in molecular analyses and data processing. We also thank the medical editor from the Division of Education for Clinical Research at the National Center for Child Health and Development for editing the final draft. 


\section{Funding}

This work was supported by a grant from the Japan Agency for Medical Research and Development (AMED) (18ek0109204h0002)

\section{Availability of data and materials}

The datasets used and/or analysed during the current study are available from the corresponding author on reasonable request.

\section{Authors' contributions}

KM designed the project, performed the molecular and data analyses, and wrote the paper. $\mathrm{MI}, \mathrm{KS}, \mathrm{SS}, \mathrm{KE}, \mathrm{KK}$, and $\mathrm{TO}$ recruited and managed the cases enrolled in this study and interpreted the cases' data from the point of view of clinical genetics. KN and $\mathrm{KH}$ supervised the bioinformatics analysis. MF and MK provided the facilities and resources for the study. MK gave the final approval of the version to be published. All authors read the manuscript, revised it critically for important intellectual content, and approved the final manuscript.

\section{Ethics approval and consent to participate}

This study was approved by the Institute Review Board Committees at the National Center for Child Health and Development (number 518) and performed after obtaining written informed consent from the parents.

\section{Consent for publication}

Consent for publication were obtained from all patients or their parents.

\section{Competing interests}

The authors declare that they have no competing interests.

\section{Publisher's Note}

Springer Nature remains neutral with regard to jurisdictional claims in published maps and institutional affiliations.

\section{Author details}

'Department of Molecular Endocrinology, National Center for Child Health and Development, 2-10-1 Ohkura, Setagaya-ku, Tokyo 157-8535, Japan. 2Department of Pediatrics, Kanazawa Medical University, Kanazawa 920-1192, Japan. ${ }^{3}$ Division of Medical Genetics, Saitama Children's Medical Center Saitama 330-8777, Japan. ${ }^{4}$ Department of Pediatrics and Neonatology, Nagoya City University Graduate School of Medical Sciences, Nagoya 467-8601, Japan. ${ }^{5}$ Enomoto Children's Clinic, Moriya 302-0127, Japan. ${ }^{6}$ Department of Pediatrics and Developmental Biology, Tokyo Medical and Dental University Graduate School, Tokyo 113-8510, Japan. Department of Maternal-Fetal Biology, National Center for Child Health and Development, Tokyo 157-8535, Japan. ${ }^{8}$ Division of Medical Genetics, Kanagawa Children's Medical Center, Yokohama 232-8555, Japan. ${ }^{9}$ Department of Pediatrics, Hamamatsu University School of Medicine, Hamamatsu 431-3192, Japan.

Received: 28 December 2018 Accepted: 14 February 2019

Published online: 28 February 2019

References

1. Horsthemke B, Wagstaff J. Mechanisms of imprinting of the Prader-Willi/ Angelman region. Am J Med Genet A. 2008;146A:2041-52. https://doi.org/ 10.1002/ajmg.a.32364.

2. Cassidy SB, Schwartz S, Miller UL, Driscoll DJ. Prader-Willi syndrome. Genet Med. 2012;14:10-26. https://doi.org/10.1038/gim.0b013e31822bead0.

3. Buiting $\mathrm{K}$, Williams $\mathrm{C}$, Horsthemke $\mathrm{B}$. Angelman syndrome-insights into a rare neurogenetic disorder. Nat Rev Neurol. 2016;12:584-93. https://doi.org/ 10.1038/nrneurol.2016.133.

4. Sahoo T, del Gaudio D, German JR, Shinawi M, Peters SU, Person RE, et al. Prader-Willi phenotype caused by paternal deficiency for the HBII-85 C/D box small nucleolar RNA cluster. Nat Genet. 2008;40:719-21. https://doi.org/ 10.1038/ng.158.

5. de Smith AJ, Purmann C, Walters RG, Ellis RJ, Holder SE, Van Haelst MM, et al. A deletion of the HBII-85 class of small nucleolar RNAs (snoRNAs) is associated with hyperphagia, obesity and hypogonadism. Hum Mol Genet. 2009;18:3257-65. https://doi.org/10.1093/hmg/ddp263.

6. Duker AL, Ballif BC, Bawle EV, Person RE, Mahadevan S, Alliman S, et al. Paternally inherited microdeletion at $15 q 11.2$ confirms a significant role of the SNORD116 C/D box snoRNA cluster in Prader-Willi syndrome. Eur J Hum Genet. 2010;18:1196-201. https://doi.org/10.1038/ejhg.2010.102.
7. Bieth E, Eddiry S, Gaston V, Lorenzini F, Buffet A, Conte Auriol F, et al. Highly restricted deletion of the SNORD116 region is implicated in Prader-Willi syndrome. Eur J Hum Genet. 2015;23:252-5. https://doi.org/10.1038/ejhg. 2014.103

8. Fontana P, Grasso M, Acquaviva F, Gennaro E, Galli ML, Falco M, et al. SNORD116 deletions cause Prader-Willi syndrome with a mild phenotype and macrocephaly. Clin Genet. 2017;92:440-3. https://doi.org/10.1111/cge.13005.

9. Buiting K. Prader-Willi syndrome and Angelman syndrome. Am J Med Genet C Semin Med Genet. 2010;154C:365-76. https://doi.org/10.1002/ajmg.c.30273.

10. Buiting K, Gross S, Lich C, Gillessen-Kaesbach G, el-Maarri O, Horsthemke B. Epimutations in Prader-Willi and Angelman syndromes: a molecular study of 136 patients with an imprinting defect. Am J Hum Genet. 2003;72:571-7. https://doi.org/10.1086/367926.

11. Joshi RS, Garg P, Zaitlen N, Lappalainen T, Watson CT, Azam N, et al. DNA methylation profiling of uniparental disomy subjects provides a map of parental epigenetic bias in the human genome. Am J Hum Genet. 2016;99: 555-66. https://doi.org/10.1016/j.ajhg.2016.06.032.

12. Court F, Tayama C, Romanelli V, Martin-Trujillo A, Iglesias-Platas I, Okamura $\mathrm{K}$, et al. Genome-wide parent-of-origin DNA methylation analysis reveals the intricacies of human imprinting and suggests a germline methylationindependent mechanism of establishment. Genome Res. 2014;24:554-69. https://doi.org/10.1101/gr.164913.113.

13. Sharp AJ, Migliavacca E, Dupre Y, Stathaki E, Sailani MR, Baumer A, et al. Methylation profiling in individuals with uniparental disomy identifies novel differentially methylated regions on chromosome 15. Genome Res. 2010;20: 1271-8. https://doi.org/10.1101/gr.108597.110.

14. Rabinovitz S, Kaufman Y, Ludwig G, Razin A, Shemer R. Mechanisms of activation of the paternally expressed genes by the Prader-Willi imprinting center in the Prader-Willi/Angelman syndromes domains. Proc Natl Acad Sc U S A. 2012;109:7403-8. https://doi.org/10.1073/pnas.1116661109.

15. Castle JC, Armour CD, Löwer M, Haynor D, Biery M, Bouzek H, et al. Digital genome-wide ncRNA expression,including SnoRNAs, across 11 human tissues using poly A-neutral amplification. PLoS One. 2010:5:e11779. https:// doi.org/10.1371/journal.pone.0011779.

16. Yin QF, Yang L, Zhang Y, Xiang JF, Wu YW, Carmichael GG, et al. Long noncoding RNAs with snoRNA ends. Mol Cell. 2012;48:219-30. https://doi. org/10.1016/j.molcel.2012.07.033.

17. Powell WT, Coulson RL, Crary FK, Wong SS, Ach RA, Tsang P, et al. A PraderWilli locus IncRNA cloud modulates diurnal genes and energy expenditure. Hum Mol Genet. 2013;22:4318-28. https://doi.org/10.1093/hmg/ddt281.

18. Coulson RL, Yasui DH, Dunaway KW, Laufer BI, Vogel Ciernia A, Zhu Y, et al. Snord116-dependent diurnal rhythm of DNA methylation in mouse cortex. Nat Commun. 2018;9:1616. https://doi.org/10.1038/s41467-018-03676-0.

19. Lewis MW, Brant JO, Kramer JM, Moss JI, Yang TP, Hansen PJ, et al. Angelman syndrome imprinting center encodes a transcriptional promoter. Proc Natl Acad Sci U S A. 2015;112:6871-5. https://doi.org/10.1073/pnas.141126111.

20. Morris TJ, Butcher LM, Feber A, Teschendorff AE, Chakravarthy AR, Wojdacz TK, et al. ChAMP: 450k Chip Analysis Methylation Pipeline. Bioinformatics. 2014;30:428-30. https://doi.org/10.1093/bioinformatics/btt684

Ready to submit your research? Choose BMC and benefit from:

- fast, convenient online submission

- thorough peer review by experienced researchers in your field

- rapid publication on acceptance

- support for research data, including large and complex data types

- gold Open Access which fosters wider collaboration and increased citations

- maximum visibility for your research: over $100 \mathrm{M}$ website views per year

At BMC, research is always in progress.

Learn more biomedcentral.com/submissions 